\title{
IMPULSO Y RESISTENCIA EN LA CREACIÓN DE CENTROS INTEGRADOS EN ARAGÓN
}

\author{
IMPULSE AND RESISTANCE IN THE CREATION \\ OF INTEGRATED CENTRES IN ARAGON
}

http://dx.doi.org/10.15304/ie.28.5484

\author{
Fernando Andrés Rubia \\ Maestro y sociólogo \\ Director de las revistas Fórum Aragón y Organización y Gestión Educativa \\ fandres61@gmail.com
}

\section{RESUMEN}

El curso 2017-18 se crearon por primera vez en Aragón los dos primeros Institutos-Escuela, con el nombre de centros integrados, a partir de la ampliación de colegios de infantil y primaria. La administración apuesta por la transformación de algunos centros existentes y la planificación en el futuro de nuevos centros con estas características como respuesta a la falta de plazas escolares de Secundaria en algunos barrios y zonas periféricas. Celebramos el centenario de la creación de los primeros Institutos-Escuela reconociendo sus aportaciones educativas innovadoras especialmente en aspectos relacionados con las transiciones de etapas. El Departamento de Educación, Cultura y Deporte del Gobierno de Aragón ha planificado la creación en los próximos años de hasta 18 centros y ha empezado a desarrollar una normativa específica para abordar su nueva estructura y organización. Ante esta apuesta escolar y este cambio de modelo de centro, analizaremos la respuesta y la reacción de los diferentes sectores de la comunidad educativa, destacando los apoyos y las principales resistencias que se presentan frente al cambio de modelo.

Palabras clave: centros integrados, instituto-escuela, Aragón

\section{ABSTRACT}

During the academic year 2017-2018, for the first time in Aragon there was created two new Schools which combined Primary and Secondary education. These schools are called Integrated Centres because they are expansions of pre-schools and primary schools. The administration is committed to transform some of the existing centres and is planning to create new centers with these features. The reasons behind this, is a response to the lack of Secondary School places in some neighbourhoods and outskirts areas. We celebrate the centenary of the first Primary School-High School recognising their innovative educational contributions, especially their integrated approach to transition between different stages. In the coming years, the department of Education, Culture and Sports of Aragon Government is planning to create up to 18 centers. It also developing a specific policy to address the new structure and organisation of these Integrated Centres. In this article, we aim to analyse the response of the different sectors in the education community, highlighting the support and the barriers arised when implementing an Integrated School.

Keywords: Integrated Centres, Primary-High School, Aragon

Recibido: 24/IX/2018. Aceptado: X/2018 


\section{INTRODUCCIÓN}

Centro integrado, instituto-escuela o colegio-instituto son los diferentes apelativos con los que se conoce un modelo educativo que integra básicamente la formación obligatoria, aunque a veces acompañado de otras etapas. Dependiendo de la comunidad autónoma se han adoptado alguno de estos términos, por ejemplo, en Aragón son centros integrados y en Cataluña, sin embargo, siguen el término acuñado a principios del siglo XX de instituto-escuela; en algunos países latinoamericanos prefieren colegio-instituto. A lo largo del texto utilizaré mayoritariamente, pero no exclusivamente, la expresión "centro integrado" priorizando el término que ha elegido la administración aragonesa. El uso de uno u otro término, como suele suceder, no es trivial. Los institutos-escuela enlazan con la tradición de renovación educativa del centro creado inicialmente a instancias de la Junta de Ampliación de Estudios e Investigaciones Científicas en 1918 y del que celebramos su centenario. Sin embargo, los centros integrados se constituyen a partir de argumentos como el uso eficiente de los recursos y en un segundo plano, educativos o pedagógicos. En todo caso, señalar también que tras las expresiones instituto-escuela y colegio-instituto, se muestra una cierta rivalidad de dos instituciones que se ordenan por orden de prioridad o de preeminencia; el término centro integrado soslaya está tensión.

Los centros integrados o institutos-escuela son una excepción en nuestro sistema educativo público, no así en los centros privados que de forma tradicional han impartido todos los niveles educativos obligatorios en el mismo centro. En Aragón, como en la mayoría de territorios del estado, durante todo el siglo XX y primera década del XXI se crearon centros educativos públicos, siguiendo un modelo basado en la separación del alumnado por etapas educativas y edades, simplificando, los colegios de primaria se completan con aulas de educación infantil y los institutos, que han pasado por diferentes épocas, especializándose, unos, en enseñanzas como el bachillerato, y otros, en la formación profesional o agrupando toda la formación secundaria, obligatoria o no, en sus aulas.

Este modelo cerrado se adapta mal a las diferentes realidades que se dan en un territorio tan diverso y complejo como es el de Aragón, con características tan acentuadas como una gran despoblación y dispersión; y a la vez, una gran concentración urbana en la ciudad de Zaragoza. Tampoco tiene en cuenta cuestiones relacionadas con la estructura de la población y su distribución, con criterios pedagógicos, o con los intereses de las familias.

Sin embargo, lo más destacado de este modelo por etapas o de especialización limitada de los centros, es su permanencia en el tiempo y sobre todo su condición de modelo incuestionable. Sorprende porque en los centros privados precisamente nos encontramos con una tradición y una configuración muy diferente: la propuesta educativa de los centros tanto concertados como exclusivamente privados abarca generalmente las etapas obligatorias a las que se añade de nuevo la etapa infantil. La oferta varía de un centro a otro, en la elección entre bachillerato o formación profesional.

Los primeros argumentos que aparecen son las razones históricas, que tienen un peso fundamental en este modelo, razones basadas en definitiva en la tradición, pero una mirada más atenta apunta también a los intereses corporativos de los diferentes grupos profesionales en los que está dividido el profesorado. Los profesionales se mantienen en agrupaciones administrativas 
diferentes, cuerpos, con unas condiciones económicas, laborales y de acceso diferenciadas y jerarquizadas. Los grupos de profesionales tienden a mantener el statu quo, porque en paralelo, a lo largo de los años se han ido distanciando y diferenciando sus culturas profesionales, si es que alguna vez estuvieron próximas. Cada una pone el acento de su actividad e incluso de su función en aspectos diferentes, una cultura considera que la pedagogía y el niño son el instrumento y el foco de su tarea respectivamente; y la otra, la materia y el profesor sustituyen a los anteriores.

El presente trabajo se enmarca dentro de los estudios de las políticas educativas en el diseño de los centros escolares, competencia de las administraciones autonómicas. En primer lugar, haremos una breve incursión en la historia de los institutos-escuela, su origen y su caracterización como centros innovadores. Veremos también de qué forma pueden contribuir los centros integrados a mitigar algunos problemas de nuestro sistema educativo. A continuación, vamos a ver las acciones que ha emprendido la administración educativa aragonesa en fechas recientes en dos aspectos fundamentales: la creación de centros integrados y la organización de los mismos. Posteriormente, indagaremos en el grado de receptividad de los diferentes sectores educativos, en especial del profesorado y de las familias. Para finalizar, en las conclusiones hablaremos de los actores y fuerzas que impulsan estos cambios y de las que ejercen resistencia.

\section{ORIGEN DEL INSTITUTO-ESCUELA E INNOVACIÓN}

Los institutos-escuela tienen su origen en la España de principios del siglo XX en los que aparecen como instituciones pioneras en la implantación de metodologías y recursos educativos procedentes de Europa, y que desaparecieron tras la guerra y la caída de la II República. En 1907 se crea la Junta de Ampliación de Estudios e Investigaciones Científicas con el fin de modernizar el sistema educativo español. Entre sus objetivos se encontraban: fomentar las relaciones internacionales en materia educativa, promover la ampliación de estudios del profesorado dentro y fuera de España, promover la investigación científica y proteger las instituciones educativas en la enseñanza secundaria y superior. Esta nueva institución era heredera de los principios de la Institución Libre de Enseñanza (ILE), y pretendía acabar con el aislamiento cultural de España, enlazando con la ciencia y la cultura europeas, y preparando a los profesionales encargados de llevar a cabo las reformas en la ciencia, la cultura y la educación.

La Junta promovió, desde un principio, la renovación de la educación española, priorizando los primeros años la formación universitaria y científica. En 1918, creó el Instituto-Escuela de Madrid, dependiente del Ministerio de Instrucción Pública pero tutelado por la Junta. Se pensó como centro formativo del profesorado, ya que, tras su paso por él, los docentes deberían extender la experiencia a centros de futura creación. El Instituto-Escuela concibió al niño como eje del proceso educativo, siguiendo los modelos de la ILE y de la Escuela Nueva, y diseñó un plan cíclico conforme a la normativa curricular, pero con autonomía para ampliarla y seleccionar al personal. Su metodología se caracterizaba por el fomento del estudio práctico y directo de la naturaleza, el debate, la lectura, la convivencia, la curiosidad y el esfuerzo motivado. Para alcanzar estos objetivos contó con medios adecuados: talleres, laboratorios, biblioteca, imprenta y campos de deporte. 
La enseñanza estaba planificada en tres cursos de Preparatoria que se ampliaron con una sección previa de infantil y seis cursos de Bachillerato. En este último nivel, los cuatro primeros años eran comunes para todo el alumnado y en los dos últimos se introducía la especialización en Ciencias o Letras. A partir del curso 1931-32 se ofreció también la posibilidad de un Bachillerato único a partir de quinto curso. El plan de estudios contemplaba más asignaturas y horas de estudio práctico que los demás institutos de la época, ya que tenían el convencimiento de que la experimentación directa favorecía la comprensión y la interiorización del conocimiento.

Se fomentaba, además, que los padres participasen en el proceso formativo. La idea era que el Instituto-Escuela de Madrid debía servir de experiencia pionera y extenderse posteriormente por todo el país. Sin embargo, hasta los años de la República (1931-1933) no se crearon otros similares en Barcelona, Málaga y Sevilla. El Instituto-Escuela de Barcelona tomó como referentes la Escuela Moderna de Ferrer i Guardia y la guía pedagógica del Instituto-Escuela de Madrid: una pedagogía activa basada en el trabajo y la experimentación del alumno. El Instituto-Escuela de Madrid fue cerrado en 1939.

En la actualidad, debemos destacar el sistema educativo catalán que cuenta con 25 InstitutosEscuela y que además los ha reconocido en la Ley de Educación de Cataluña (2009), en su artículo 75 aparecen definidos como "los centros públicos que, entre otras enseñanzas de régimen general, imparten educación primaria y educación secundaria”.

Por último, si lanzamos la mirada a nuestro entorno europeo encontramos los dos modelos: por un lado, los llamados sistemas de gestión diferenciada, como el caso español, y por otro, los sistemas de gestión integrada de la educación primaria y secundaria. La gestión integrada, que ahora nos interesa, predomina en los países nórdicos (Dinamarca, Finlandia, Islandia, Noruega y Suecia) y en algunos del este (Bulgaria, Croacia, Eslovaquia, Eslovenia, Estonia, Hungría, Lituania, Republica Checa, y Turquía).

Desde la reforma que introdujo la LOGSE en 1990, ha surgido una cierta preocupación por el paso de Primaria a Secundaria como un momento de cambio delicado. De hecho, se pone el acento en las transiciones entre estas dos etapas para facilitar la adaptación del alumnado al cambio. A pesar de todas las medidas que puedan adoptar los institutos, y hay que reconocer un esfuerzo, los alumnos se enfrentan a un cambio sustancial caracterizado por la dispersión de materias, con un profesor por asignatura; una relajación de la acción tutorial pues el profesor tutor apenas pasa unas horas semanales en el aula; un desplazamiento del protagonismo escolar del alumno a la materia; y una mayor importancia de las normas y de la disciplina, pero a la vez una mayor autonomía y exigencia de responsabilidad al alumno, a pesar de su inmadurez. Todo ello hace que el cambio de centro se convierta en un cambio brusco que exige a los alumnos un complejo proceso de adaptación.

\section{LOS CENTROS INTEGRADOS FRENTE A ALGUNOS PROBLEMAS DEL SISTEMA EDUCATIVO}

En los últimos años del Instituto-Escuela, la dirección de la Junta de Ampliación de Estudios mostró su insatisfacción con los resultados obtenidos y señaló como causas a corregir la 
insuficiencia de las instalaciones, una matrícula y un programa demasiado recargado, la falta de preparación y motivación del profesorado y la falta de colaboración entre las secciones Preparatoria y Secundaria.

Si trasladamos a la actualidad estas preocupaciones vemos que algunas de las quejas se repiten hoy, en cualquier centro, como la escasez de recursos o unas instalaciones inadecuadas; y en cuanto al programa, si nos atenemos al currículo son muchas las voces que hablan de una sobrecarga y de la necesidad de acotarlo ${ }^{1}$. En cuanto a la preparación y motivación del profesorado también han surgido propuestas que pretenden corregir estos problemas, la más reciente la adopción de un modelo similar al MIR. También se insiste en la necesidad de mejorar los procesos de selección previos a la incorporación a los estudios, la formación inicial o los sistemas de acceso a la docencia. No son temas a los que los centros integrados puedan hacer aportaciones, porque están lejos de sus responsabilidades, pero sí que es interesante entretenernos en la última cuestión, la coordinación entre etapas, específicamente entre Primaria y Secundaria, que de forma conjunta constituyen la educación obligatoria.

Una de las dificultades a las que se enfrenta el alumnado de la escuela pública es la falta de continuidad en los procesos educativos y una infructuosa transición entre etapas. El cambio afecta aspectos como los espacios, horarios, áreas, profesorado, organización, estructura, normas... Según cuenta Pérez-Díaz et al. (2001) los cambios de centro se producen especialmente en la enseñanza pública con el paso de la Primaria a la Secundaria, es decir, son cambios forzados por el propio sistema ya que las familias se muestran razonablemente satisfechas con el funcionamiento del centro de sus hijos.

Por ello parece necesaria la colaboración entre colegios e institutos y evitar así un cambio problemático con consecuencias no deseadas, sin embargo, la realidad nos muestra que esta coordinación es restringida y poco sistematizada. En primer lugar, en el tiempo, ya que básicamente maestros y profesores sólo se reúnen a final de curso; y en segundo lugar en cuanto al contenido, que se limita habitualmente a un traspaso de información sobre el rendimiento y comportamiento de los alumnos; además, se completa con una presentación de las características y del funcionamiento de sus centros a los padres de los futuros alumnos. Por supuesto, no se plantea un proyecto educativo común o una aproximación en sus proyectos o unas normas de convivencia compartidas o adaptadas temporalmente, ni siquiera una colaboración estable con actividades paralelas.

Para entender mejor las diferencias que se producen entre los centros nos servirá el concepto de cultura escolar que nos permite distinguir entre dos culturas profesionales muy diferentes: la procedente de primaria y la de secundaria. Antonio Viñao (2002) considera que la cultura escolar está constituida por "un conjunto de teorías, ideas, principios, normas, pautas, rituales, inercias, hábitos y prácticas (formas de hacer y pensar, mentalidades y comportamientos) sedimentadas a lo largo del tiempo en forma de tradiciones, regularidades y reglas de juego no puestas en entredicho, y compartidas por sus actores, en el seno de las instituciones educativas". Existen rasgos culturales

Véase por ejemplo Gimeno Sacristán, 2018. 
estables y persistentes que caracterizan y distinguen los modos de actuar entre los centros de Primaria y los de Secundaria. Estas diferencias se reflejan en su estructura académica y disciplinar, en su organización interna, en la forma de impartir las clases o en la metodología didáctica, también en la manera de relacionarse con los alumnos, con los compañeros y con las familias de los alumnos. Esto explica, en buena parte, las dificultades que se les plantean a los alumnos en el tránsito de una etapa a otra y las diferencias que surgen cuando se integran en un mismo centro profesores de uno y otro nivel.

Fernández Enguita et al. (2010) intentan explicar los motivos del fracaso y abandono escolar desde la vivencia de la escolaridad y descubren que son los propios afectados los que reconocen que los momentos de cambio de etapa resultan críticos. Muy especialmente si el cambio de etapa va acompañado de un cambio de centro. Por supuesto, el momento más señalado en la escuela pública es el paso de Primaria a Secundaria, el paso del colegio al instituto. Las razones que destacan es que supone un cambio importante en la relación entre profesores y alumnos. Al cambiar de centro y pasar al instituto se produce un incremento en el número de alumnos por clase y sobre todo se multiplican los profesores por grupo, lo que explica, en parte, esa falta de seguimiento y acompañamiento personal.

¿Qué ventajas pueden ofrecer los centros integrados? Pueden corregir algunas de las consecuencias apuntadas: ahorra al alumnado y a las familias el cambio y el consiguiente proceso de adaptación y facilita el arraigo escolar, favorece la aproximación entre las culturas profesionales al convivir dentro del mismo ámbito organizativo, permite una mayor coherencia educativa en las etapas de formación básica, favorece la continuidad en la implantación y uso de las TIC, facilita el clima escolar y permite el recurso a la responsabilidad de los alumnos mayores frente a los más pequeños. El modelo integrado permite abordar estas dificultades de los alumnos como un proceso sin discontinuidades, el cambio de etapa forma parte de la normalidad del centro y no tiene por qué contribuir a la desestabilización del alumno. Permite, además, el acercamiento de las dos culturas profesionales.

\section{LOS PRIMEROS PASOS EN LA PUESTAEN MARCHA DE LOS CENTROS INTEGRADOS ARAGONESES}

En el curso 2017-2018 se inició el proceso de creación de Institutos-Escuela en Aragón de forma testimonial con la ampliación de las etapas educativas en dos colegios, uno urbano, La Jota (Zaragoza) y otro rural en Ayerbe (Huesca); es decir, a partir de la transformación de dos centros ya existentes de Infantil y Primaria. En 2018, el Departamento de Educación, Cultura y Deporte del Gobierno de Aragón ha aprobado la transformación de algunos colegios más en centros integrados y la construcción de otros nuevos que nacerán como centros integrados. Con la información obtenida del Departamento hemos elaborado el siguiente cuadro que da idea de la dimensión de la propuesta: 


\begin{tabular}{|c|c|c|c|}
\hline Curso & $\begin{array}{l}\text { Centro Público } \\
\text { Integrado }\end{array}$ & Localidad & Enseñanzas \\
\hline \multirow[t]{2}{*}{$2017-18$} & La Jota & Zaragoza & EI, EP, EBO y ESO \\
\hline & Ramón y Cajal & Ayerbe (Huesca) & EI, EP y ESO \\
\hline \multirow[t]{9}{*}{ 2018-19 } & El Espartidero & Zaragoza & $\mathrm{EI}, \mathrm{EP}, \mathrm{ESO}\left(1^{\circ}\right)$ \\
\hline & Galo Ponte & $\begin{array}{l}\text { San Mateo de } \\
\text { Gállego (Zaragoza) }\end{array}$ & EI, EP, ESO $\left(1^{\circ}\right)$ \\
\hline & Julio Verne & Zaragoza & $\begin{array}{l}\text { EI, EP (hasta } 4^{\circ} \text {, empieza con } \\
\text { primaria incompleta) }\end{array}$ \\
\hline & Miralbueno & Zaragoza & $\begin{array}{l}\text { EI, EP y ESO, Como es nuevo, } \\
\text { el alumnado lo trasladan } \\
\text { provisionalmente al Julio Verne }\end{array}$ \\
\hline & Parque Goya & Zaragoza & EI, EP y ESO $\left(1^{\circ}\right)$ \\
\hline & Rosales del Canal & Zaragoza & EI, EP y $\operatorname{ESO}\left(1^{\circ}\right)$ \\
\hline & San Jorge & Zaragoza & $\begin{array}{l}\text { EI, EP (hasta } 4^{\circ} \text {, empieza con } \\
\text { primaria incompleta) }\end{array}$ \\
\hline & Val de Atalaya & $\begin{array}{l}\text { María de Huerva } \\
\text { (Zaragoza) }\end{array}$ & EI, EP y ESO \\
\hline & Zaragoza Sur & Zaragoza & $\begin{array}{l}\text { EI, EP (hasta } 2^{\circ} \text {, empieza con } \\
\text { primaria incompleta) }\end{array}$ \\
\hline \multirow[t]{2}{*}{$2019-20$} & Agustina de Aragón & Zaragoza & \\
\hline & Castillo de Qadrit & Cadrete (Zaragoza) & $\begin{array}{l}\text { Centro de primaria que } \\
\text { comienza con } 1^{\circ} \mathrm{ESO}\end{array}$ \\
\hline \multirow[t]{5}{*}{ En construcción } & Valdespartera 3 & Zaragoza & EI, EP, ESO y Bachillerato \\
\hline & $\begin{array}{l}\text { Valdespartera } 4 \text { (ahora } \\
\text { CPI Soledad Puértolas) }\end{array}$ & Zaragoza & EI, EP, ESO y Bachillerato \\
\hline & Valdespartera 5 & Zaragoza & EI, EP, ESO y Bachillerato \\
\hline & Arcosur & Zaragoza & EI, EP, ESO y Bachillerato \\
\hline & Parque Venecia & Zaragoza & EI, EP, ESO y Bachillerato \\
\hline
\end{tabular}

Tabla 1. Planificación de Centros Integrados de la C. A. de Aragón. Fuente: elaboración propia.

La cuestión fundamental es entender cuáles han sido los motivos que han llevado a la administración aragonesa a este cambio en la política tradicional de centros. Como reconoció el secretario general técnico del Departamento de Educación, Cultura y Deporte en una comparecencia ${ }^{2}$ ante la Comisión de Educación de las Cortes de Aragón, el nuevo modelo de centros integrados responde a dos objetivos: uno pedagógico, que permite nuevas estructuras de coordinación

2 Ver http://www.aragonhoy.net/index.php/mod.noticias/mem.detalle/id.215918 
pedagógica y de gestión educativa; y otro funcional, que da nuevos usos a los espacios y recursos educativos y modifica la gestión económica y presupuestaria para atender la creciente demanda de plazas en Secundaria.

En realidad, el problema al que han tenido que hacer frente es a la demanda de plazas escolares en determinadas zonas de expansión en Infantil y Primaria, y que ahora se han trasladado a Secundaria. La falta de previsión y planificación, y la ausencia de inversión del anterior equipo de gobierno, supuso que al inicio de la legislatura se produjera un importante déficit de plazas escolares públicas, que obligó a cerca de un millar de alumnos a desplazarse y escolarizarse lejos de su domicilio. La actual administración, ante la dificultad de dar solución a un problema que promete extenderse en un futuro próximo, ha decidido que en los casos en los que existe la posibilidad de adaptación de los colegios, ampliar su oferta educativa; y en los de nueva creación, adaptar directamente las nuevas infraestructuras educativas a la demanda.

Este cambio en política de creación de centros ha sido justificado por el Departamento de Educación como respuesta a las necesidades demográficas, optimizando la inversión pública, abaratando costes y aprovechando las instalaciones de los colegios en cuyas zonas se ha ido perdiendo alumnado de Educación Primaria mientras que se ha incrementado el de Secundaria. Se trata entonces de una apuesta indirecta por los centros integrados, ya que, entre los argumentos esgrimidos, el de mayor peso es la falta de plazas escolares y la dificultad de atender la futura demanda, es decir, responde a criterios economicistas y de eficiencia.

No por ello, debemos dejar de valorar que el nuevo modelo de centros integrados potencia la coordinación entre las diferentes etapas, especialmente entre Primaria y Secundaria, así como la coordinación de proyectos educativos. Pero, lo cierto es que los argumentos pedagógicos quedan en un segundo plano, debemos entender que no estamos ante un proceso de innovación educativa o de cambio razonado de criterios pedagógicos y metodológicos, es más, el objetivo principal no es favorecer las transiciones entre las etapas educativas y mejorar resultados.

\section{UN PRIMER ACERCAMIENTO NORMATIVO: LA ORGANIZACIÓN DE LOS NUEVOS CENTROS}

Una vez adoptada la decisión política de creación de los centros integrados, es fundamental el diseño de los nuevos centros y considerar ahora sí qué criterios pedagógicos se tienen en cuenta en su estructura y organización.

La principal dificultad sin duda es la adaptación de la organización de los nuevos centros ya que colegios e institutos difieren en cuanto a modelos organizativos. Para ello la ORDEN ECD/783/2017, de 8 de junio, regula por primera vez las condiciones de organización y funcionamiento del Centro Público Integrado La Jota de Zaragoza a partir del curso 2017-2018 y define como Centro Público Integrado "aquel que imparta, al menos, la educación básica en su totalidad".

La Orden establece la organización de los nuevos centros en base a los siguientes principios generales: La unidad del centro, no se trata de un conglomerado con secciones independientes, 
sino de un único centro con un proyecto educativo común que mantiene una estructura similar a los centros de Primaria y Secundaria en cuanto a los órganos unipersonales (dirección, jefaturas de estudios especializadas y secretaría) y colegiados (Consejo Escolar y Claustro de Profesores). Se asegura la unidad del centro educativo y queda abierta la posibilidad de colaboración entre etapas educativas para dar así continuidad a los procesos educativos favoreciendo las transiciones.

Pone el acento en la coordinación mediante la creación de diferentes equipos docentes (equipos de transición, equipos didácticos en Primaria y de curso en la ESO), el equipo de orientación (en el que destaca las tareas con el alumnado con necesidades de apoyo y la convivencia), la Comisión Pedagógica de Coordinadores (CPC), nueva denominación de la Comisión de Coordinación Pedagógica (CCP), el Equipo de Actividades, Recursos y Comunicación o el Equipo de Formación, Innovación y Proyectos.

El equipo de transición supone el reconocimiento de las dificultades en el cambio de etapa, ya señaladas, poniendo el énfasis en la coordinación del profesorado de las dos etapas. Es importante constatar que la creación de los nuevos equipos supone la desaparición de los clásicos departamentos por áreas de Secundaria. Además, entre las preocupaciones destaca la convivencia entre alumnado de una gran amplitud de edades (entre los 3 y los 16 años, ampliables hasta los 18) corresponsabilizando a los docentes, pero asignando la tarea específica al equipo de orientación y creando la figura de un responsable de centro.

Entre las tareas asignadas a los equipos destaca la preocupación por el desarrollo de actividades de ocio, tiempo libre y deporte en horario extraescolar que supone una aproximación entre dos ideas alejadas hasta ahora como son los tiempos escolares y los tiempos educativos; la importancia que asigna a la comunicación; y el impulso a la formación del profesorado, la innovación educativa y la puesta en marcha de proyectos de centro. Otra de las preocupaciones es la de los horarios y cómo conjugar realidades diferentes, se apela a mantener un criterio homogeneizador y al uso eficiente de los recursos del centro.

¿Qué se echa en falta? Sobre todo, muchos aspectos relacionados con el profesorado que debe asumir el reto de convertir el centro integrado en un modelo organizativo eficaz. Concretamente sobre los horarios del profesorado, la orden solo hace alusión a los cupos, olvidando cuestiones fundamentales que les afectan derivadas de la supresión de los departamentos. Parece necesaria una especificación de las condiciones en las que se asumen las distintas coordinaciones de equipos, sobre todo, para evitar agravios comparativos con los docentes de colegios e institutos. No olvidemos, además, que será el profesorado el que deberá implementar estas medidas, es importante implicarle, conseguir su colaboración e incluso lograr su complicidad para evitar así que una actitud negativa y de rechazo dificulte todo el proceso.

Veamos ahora con más detalle los aspectos organizativos que contempla la Orden:

- El Proyecto Educativo de Centro (PEC) será unitario y en su elaboración participará toda la Comunidad Educativa. Además, el proyecto se podrá organizar a través de proyectos de trabajo tanto interdisciplinares como interetapas, abriendo la puerta a la colaboración inter e intra nivelar. 
- Los órganos de gobierno unipersonales se mantienen: director, jefe de estudios (que ahora será de Primaria) y secretario, pero incorpora un jefe de estudios de Educación Secundaria. Sorprendentemente se olvidan de la etapa de Infantil, aunque razonablemente será asumido por el de Primaria. De los órganos colegiados mantiene el Consejo Escolar y el Claustro de Profesores, e incorpora al nuevo jefe de estudios al Consejo.

- Equipos docentes: Cada equipo docente estará formado por los maestros y profesores que impartan áreas de conocimiento en cada uno de los grupos de los niveles educativos. Cada equipo será coordinado por el tutor de referencia del grupo clase y los tutores serán los representantes de los equipos docentes en la Comisión Pedagógica de Coordinadores. Establece también la obligación de reunirse con una periodicidad de al menos una vez al mes. Hay tres tipos de equipos docentes:

- Equipo de transición del alumnado de la etapa de Educación Primaria a la de Educación Secundaria Obligatoria, compuesto por los maestros que impartan sexto de Educación Primaria y los profesores que impartan primero de ESO. Este equipo será coordinado conjuntamente por las Jefaturas de Estudios.

- Equipos didácticos en Educación Primaria.

- Equipos de curso en Educación Secundaria Obligatoria.

- Equipo de Orientación: Estará formado por el orientador que será su coordinador y el profesorado que realice tareas de atención al Alumnado con Necesidades Específicas de Apoyo Educativo. Este equipo desarrollará las funciones asignadas al equipo de orientación educativa y psicopedagógica de Infantil y Primaria y al departamento de orientación en Secundaria establecidas en la normativa general. Será responsable de la mejora de la convivencia y la prevención de conflictos, así como de la resolución de los que surjan. Todos los equipos docentes en las reuniones mensuales atenderán el tema de la convivencia de forma específica. Se nombra un responsable de convivencia escolar que colabora con el orientador en la coordinación, que será también miembro del equipo de Orientación y que participará en la Comisión Pedagógica de Coordinadores.

- Equipo de actividades, recursos y comunicación. Siguiendo el modelo de los institutos, incorpora un nuevo equipo, con tareas complementarias dando protagonismo a aspectos de ocio y tiempo libre, de carácter cultural y deportivo. Tendrán al menos una reunión mensual con las jefaturas de estudios. Entre sus funciones destacan las de coordinar las actividades escolares complementarias y las actividades extraescolares; desarrollar, actualizar y coordinar los medios de comunicación de la comunidad educativa; desarrollar programas europeos; impulsar y coordinar actividades culturales; e impulsar y coordinar actividades deportivas.

- Equipo de formación, innovación y proyectos. Se trata de dar protagonismo e incentivar los procesos de cambio y transformación educativa. Está formado por el coordinador de Formación e Innovación, que será el coordinador del equipo; el responsable de medios informáticos y audiovisuales; y el responsable de la biblioteca escolar.

En función de los proyectos educativos se podrán autorizar otros equipos cuando supongan un cambio metodológico y afecte a Primaria y Secundaria y a varias materias. Estará formado por 
todo el profesorado que participe y su coordinador será miembro de la Comisión Pedagógica de Coordinadores. Tendrán al menos una reunión mensual con las jefaturas de estudios.

En cuanto a la composición, organización y competencias de la CPC, formarán parte de la Comisión además del director y los jefes de estudios; el orientador; el responsable de convivencia escolar; los coordinadores de equipo didáctico; los coordinadores de curso; el coordinador del equipo de actividades, recursos y comunicación; el coordinador del equipo de formación, innovación y proyectos; los coordinadores de otros equipos; y otros responsables designados por el centro. La composición, organización y competencias de los miembros de la CPC serán las mismas atribuidas a la CCP.

El horario de todo el alumnado será lo más homogéneo posible, respetando las peculiaridades de cada etapa educativa y facilitando una mejor organización de la oferta educativa, el mayor rendimiento de los alumnos según su edad y el mejor aprovechamiento de los espacios y recursos del centro.

El horario de los docentes no se especifica, pero establece que el centro contará con un cupo de horas para la dedicación a las responsabilidades de equipo directivo y para el desarrollo de la coordinación de equipos y responsabilidad de proyectos.

Establece que el Departamento y los ayuntamientos firmarán convenios de colaboración para hacer frente a la conservación, mantenimiento y vigilancia de los edificios escolares en los que se ubican los centros integrados. Se trata de una dificultad añadida ya que el mantenimiento de los edificios y entorno escolar variaban según el nivel educativo: los colegios de Infantil y Primaria son responsabilidad de los ayuntamientos que corren con los gastos de sostenimiento, calefacción, limpieza y aportan el personal de conserjería; mientras que los institutos cuentan con presupuesto propio para estos gastos.

De las decisiones adoptadas por la administración, y que hemos visto hasta ahora, podemos entender que la apuesta por los centros integrados, aunque haya estado condicionada por la situación dejada por el anterior equipo de gobierno, apuesta por un modelo de cambio educativo, no solo de centro.

\section{EL PROFESORADO}

Para conocer la posición del profesorado, un colectivo diverso y complejo en el que es difícil encontrar unanimidades, partiremos de las manifestaciones de los sindicatos ${ }^{3}$ representativos

3 La información ha sido obtenida a través de las páginas webs de los sindicatos y específicamente de los siguientes enlaces: https://www.csif.es/contenido/aragon/educacion/249256; https://www.csif.es/contenido/ aragon/educacion/246427; https://www.csif.es/contenido/aragon/educacion/248613; https://www. cgtaragon.org/fedens/mesa-sectorial-19-de-abril-2018; http://www.stea.es/comunicados/18-04-24\%20 MESA\%20SECTORIAL\%2019-4-18.pdf; $\quad$ http://www.ccooeducaragon.com/ensenanzaaragon/Tu_ sector:Ensenanza_Publica:Inicio:1034847--Centros_Integrados_de_Educacion_Infantil,_Primaria_y_ Secundaria_Obligatoria. 
recogiendo la opinión de cuatro de ellos. Todos coinciden en que los centros integrados por transformación no son más que una solución rápida e improvisada a un grave problema de planificación de las administraciones educativas, que no tuvieron en cuenta la previsión de natalidad, su evolución y la construcción de nuevos centros de Educación Secundaria. Los sindicatos piden una planificación de futuro para que no haya nuevos problemas de organización de espacios como los actuales y que las medidas que se tomen no se hagan en detrimento de las condiciones laborales del profesorado. Algunos opinan que la creación de centros integrados requeriría un debate más profundo dentro de la comunidad educativa.

Algunos sindicatos, ven bien el planteamiento de los centros integrados en áreas rurales para evitar el desplazamiento de los alumnos a estudiar lejos de sus casas a edades tempranas, también en algunas zonas urbanas con alguna problemática específica pero no apoyan que sea el modelo a implantar en todos los centros de nueva creación. Para otros, la transformación de colegios en centros integrados es una solución razonable ante la falta de plazas de Secundaria, pero no comparten la forma cómo se está llevando a cabo. Por último, alguno cree que la alternativa no pasa por el centro integrado y apuesta por otras opciones organizativas para aprovechar los espacios como crear dos centros en el mismo espacio, o un centro, pero con dos estructuras organizativas diferentes, es decir, manteniendo de forma separada colegio e instituto.

También muestran su malestar por la provisionalidad de las medidas organizativas a la hora de regular el funcionamiento de los centros integrados y la falta de negociación, reclamando un Reglamento Orgánico de Centro y unas Instrucciones de Organización y Funcionamiento. Las actuales no recogen las nuevas responsabilidades, como los coordinadores de Equipo, lo que impide que sus funciones sean retribuidas, objeto de puntuación, o cuenten con las atribuciones horarias necesarias para desempeñarlas. Tampoco quedan establecidos aspectos concretos como la organización de las guardias o sustituciones cuando falta un docente, teniendo en cuenta las etapas educativas; o la organización de la atención a la diversidad en un alumnado en edades tan dispares.

Alguno incluso propone que existan complementos económicos específicos para los profesores y cargos directivos de los centros integrados por el esfuerzo extra que supone. Además, critican que, en los centros piloto, los coordinadores no han recibido ningún complemento económico, ni valoración de méritos para el concurso de traslados.

Algunos sindicatos se muestran preocupados por la desaparición de los Departamentos de Secundaria. Si bien consideran buena la mejora de la coordinación horizontal en Secundaria (Equipos de curso) dotándola del tiempo necesario; opinan que eliminar la coordinación departamental acarreará problemas en el futuro.

Otra preocupación se dirige hacia la habilitación de espacios, aulas, laboratorios, talleres, aulas específicas... Algunos sindicatos piensan que en algunos de los centros integrados tendrán problemas de espacio cuando se traslade alumnado de los cuatro cursos de la ESO. También les preocupa la adecuación y uso de comedores y patios de recreo. 


\section{LA POSICIÓN DE LAS FAMILIAS}

Contrariamente a lo que se dice, las familias hacen uso de la escuela, pero apenas participan en su configuración, en su organización o en su gestión. En las últimas décadas se ha producido una apropiación de la enseñanza por parte del profesorado en el marco de un discurso basado en la profesionalización. Como dice Latapí (1997) las familias a quienes sí interesa la educación por su propia naturaleza, son fuerzas potenciales de renovación educativa pero no pueden actuar si aquellos que se han apoderado del ámbito escolar les cierran las puertas. Hace tiempo que sabemos del mal funcionamiento de los Consejos Escolares, con una sobrerrepresentación del profesorado en relación al resto de los sectores de la comunidad educativa; habiendo sido concebido como el principal órgano de gobierno ha acabado convirtiéndose en un mero espectador, burocratizado, en el que no se abordan debates sobre temas de relieve. La modificación de la LOE en 2013 además redujo las competencias del Consejo Escolar que pasa a ser un órgano consultivo y traslada gran parte de sus competencias a la dirección de los centros.

La decisión más importante que adoptan las familias en relación con la educación de sus hijos es la elección de centro a los tres años. Un interesante estudio realizado en la ciudad de Barcelona por Alegre et al. (2010) mostraba que dos son los criterios destacados por los padres para tomar la decisión: la proximidad del centro y el proyecto educativo. La posibilidad de seguir estudios de secundaria es la quinta opción, señalada por un $22,4 \%$ de las familias, aunque aumenta hasta el 24,2\% si consideramos sólo a las familias de origen español. Es decir, que casi una de cada cuatro familias, en principio, no considera la escuela pública como opción por no ofrecer una oferta integrada. Más adelante apuntaba además que: "El motivo principal que se aduce a la hora de justificar la apuesta por la elección de escuelas concertadas remite a la valoración de la continuidad entre primaria y secundaria".

En Aragón, la posición de la Federación de AMPA, FAPAR ${ }^{4}$, queda recogida en un documento publicado en 2006 que dice textualmente: "Centros integrados (3-18 años) como garantía de continuidad en un mismo centro a las familias a lo largo de toda su vida escolar. A las familias les da gran tranquilidad saber que sus hijos pueden iniciar su etapa de formación académica obligatoria en un lugar determinado y que pueden continuar en el mismo hasta concluirla. Desde este punto de vista nos parece acertada la idea de construir centros unitarios en aquellas zonas o localidades en las que sea posible y beneficioso. Por otra parte, la unificación en un solo centro de todas las etapas obligatorias, incluido el segundo ciclo de Educación Infantil, facilitaría la coordinación entre las distintas etapas, lo que redundaría en beneficio del funcionamiento de los mismos".

De nuevo encontramos argumentos favorables, que coinciden con los técnicos, desde FAPAR 5 consideran que muchos padres entienden que los niños son muy pequeños para cambiar del colegio al instituto con 12 años o menos. Pasar al instituto implica cambiar de espacio, de amigos, de modelo

http://www.educaragon.org/files/Federaci\%C3\%B3n\%20de\%20Asociaciones\%20de\%20Padres\%20y\%20 Madres\%20de\%20Alumnos\%20-\%20FAPAR.pdf

5 http://arainfo.org/fapar-celebra-el-anuncio-del-plan-de-infraestructuras-educativas-publicas/ 
y de metodología. Para FAPAR los centros integrados son una buena solución tanto para la falta de plazas en los institutos como para dar respuesta a esta demanda de las familias.

En cuanto a las AMPA de los centros se muestran satisfechos porque sus hijos puedan continuar en el mismo centro y sus preocupaciones, expresadas a través de los medios de comunicación, se dirigen hacia las medidas provisionales y los recursos. Por ejemplo, en el colegio Miralbueno, el alumnado tendrá que desplazarse a las aulas puente que se abrirán en el Julio Verne. La falta de espacio suficiente y adecuado preocupa en el Val de Atalaya de María de Huerva ya que la ampliación del edificio no estará para el curso que viene. Los padres de Rosales de Canal también cuestionan que se tengan que perder aulas comunes. Otras preocupaciones se dirigen hacia el uso del comedor escolar por parte del alumnado de Secundaria. En el CIP de Ayerbe cuentan con comedor los chicos con beca de transporte, pero no el resto, por eso piden que estén abiertos también para el alumnado de Secundaria ${ }^{6}$. Las familias del CIP La Jota se quejan de la falta de un plan de inversiones. En El Espartidero hay que adaptar el aula de música, poner en marcha dos laboratorios y dotar al cole de medios informáticos.

\section{DISCUSIÓN Y CONCLUSIONES: IMPULSO Y RESISTENCIA}

Como hemos visto se trata de un modelo incipiente, nos encontramos ante los primeros pasos en su puesta en funcionamiento, aunque con un plan ambicioso. Por eso las conclusiones solo pueden ser provisionales y será el paso del tiempo y la implementación la que nos diga si logra asentarse como modelo alternativo al tradicional de gestión diferenciada o deja de contar con el apoyo de las mayorías políticas y se convierte en una prueba temporal y anecdótica. Si distinguimos entre los diferentes actores colectivos sería difícil encontrar consensos, pero de lo expuesto podemos deducir que en el caso de la comunidad autónoma de Aragón la administración ha encontrado en los centros integrados la solución a un grave problema de falta de plazas escolares en Secundaria. Quiere esto decir que la actual administración se ha convertido en su principal impulsor, aunque los centros integrados no aparecían como estrategia de escolarización en su programa electoral.

Es por ello, que como se le ha criticado, ha tomado las medidas de forma precipitada, obligada por la necesidad de ofrecer plazas escolares de Secundaria en determinados barrios y zonas periurbanas de Zaragoza. Esta precipitación le ha llevado a no tener previstos aspectos relevantes relacionados con el profesorado y con los recursos. Teniendo en cuenta el papel fundamental que va a jugar el profesorado en la implementación del proceso, se corre el riesgo de que un profesorado descontento y receloso dificulte su culminación.

Las familias se encuentran entre los sectores de la comunidad educativa más favorables a la implantación y por tanto impulsores de los centros integrados. Como hemos visto, las familias entienden como un beneficio para sus hijos la continuidad en el mismo centro de su recorrido escolar al menos en las dos etapas obligatorias. Hasta ahora, entendemos que la experiencia acaba de

6 https://www.heraldo.es/noticias/aragon/2018/04/02/piden-que-comedor-los-centros-integrados-abrasecundaria-1233020-300.html 
empezar, solo el futuro dirá si la decisión ha contado con los suficientes apoyos, pero sus principales demandas están relacionadas con los recursos que permiten una buena adaptación de los centros.

En cuanto al profesorado no parece que entre los centros en transformación hayan surgido grandes impedimentos, pero lo cierto es que la actitud de los sindicatos más representativos de los docentes de los centros públicos es de desconfianza y vigilancia. Solo un sindicato expresa su rechazo al modelo y propone alternativas a los centros integrados, en general predomina la actitud negociadora para resolver las cuestiones pendientes. Desde los sindicatos entienden que hay muchos aspectos por corregir y es urgente la elaboración de un Reglamento Orgánico de Centro y unas Instrucciones de Organización y Funcionamiento. La aprobación de un Reglamento y unas Instrucciones específicas para los centros integrados allanarían el camino al regular aspectos pendientes y sobre todo reduciría las desconfianzas entre los profesionales.

Aunque los sindicatos de docentes se encontrarían entre los sectores de la comunidad educativa más resistente al cambio porque han sido los que más dificultades han puesto, entendemos sin embargo que su actitud negociadora les aleja de posiciones de rechazo y permite que mediante el trabajo conjunto se encuentren soluciones que favorezcan su implantación.

En definitiva, el sistema educativo aragonés incorpora un nuevo modelo de centros escolares con importantes expectativas, pero con demasiada improvisación. La importancia de esta experiencia nos mantendrá atentos a su desarrollo y resultados.

\section{REFERENCIAS BIBLIOGRÁFICAS}

Alegre, M. À. et al. (2010). Les familias davant l'elecció escolar. Dilemes i desigualtats en la tria de centre a la ciutat de Barcelona. Barcelona: Fundació Jaume Bofill.

Andrés, F. (2011). Los centros públicos integrados de enseñanza obligatoria: un territorio inexplorado. Revista Fórum Aragón, 3, pp. 33-37.

Fernández Enguita, M. et al. (2010). Fracaso y abandono escolar en España. Barcelona: Fundación La Caixa.

Gimeno Sacristán, J. (coord.) (2018). Cambiar los contenidos, cambiar la educación. Madrid: Morata.

Latapí, P. (1997). La participación social en la educación. En J. Ezpeleta, S. Schmelkes y M. Corenstein (Coords.), Investigación educativa, gestión y participación social, Tomo 2. México: COMIE-DIE.

Martínez, M. y Pinya, C. (2015). La transición primaria-secundaria en los Institutos-Escuela en Catalunya. Revista Electrónica Interuniversitaria de Formación del Profesorado, 18(2), 57-72. DOI: http://dx.doi.org/10.6018/reifop.18.2.219141

Ontañón, E. (2007). El Instituto-Escuela, una experiencia educativa ejemplar. Revista Circunstancia, año $\mathrm{V}, 14$.

Pérez- Díaz, V. et al. (2001). La familia española ante la educación de sus hijos. Barcelona: Fundación La Caixa.

Viñao, A. (2002). Sistemas educativos, culturas escolares y reformas. Madrid: Morata. 